\title{
Die Betreuung von Kleinkindern im westeuropäischen Vergleich
}

\author{
In Deutschland wurde Anfang August 2013 eine Betreuungsgarantie für Kleinkinder \\ eingeführt. Seitdem haben alle Eltern mit Kindern ab dem vollendeten ersten Lebensjahr \\ einen Anspruch auf Teilzeitbetreuung in einer Kindertagesstätte oder in öffentlich \\ geförderter Kindertagespflege. Ein solcher Rechtsanspruch besteht für diese Altersgruppe \\ sonst nur in den skandinavischen Wohlfahrtsstaaten, die für ihre sozialen Dienstleis- \\ tungen bekannt sind. Im Folgenden geben wir einen Überblick zur Lage der Kleinkind- \\ betreuung in Westeuropa.
}

ERIC SEILS, MATTHIAS KLEIN

\section{Einleitung}

Während Deutschland mit der jüngst gegebenen Betreuungsgarantie scheinbar zu den führenden Sozialstaaten in Sachen Kinderbetreuung aufschließt, ist andererseits klar, dass die tatsächlich vorhandene Infrastruktur in der Bundesrepublik nicht ausreicht, um den Rechtsanspruch flächendeckend umsetzen zu können. Außerdem bezieht sich die Garantie im Falle der Bundesrepublik nur auf eine Halbtagsbetreuung, während in den nordischen Ländern zumeist eine Vollzeitbetreuung geboten wird.

Vor diesem Hintergrund wirft der vorliegende Beitrag einen Blick auf die Entwicklung der formalen Kleinkindbetreuung in 17 westeuropäischen Ländern: Welche historischen Ursprünge hat die Kinderbetreuung in Westeuropa? Wie hat sich das Volumen der öffentlichen Kleinkindbetreuung entwickelt, d.h. welcher Anteil der Kleinkinder wird außerhäuslich betreut und wie haben sich die täglichen Betreuungszeiten entwickelt? Ist ein bedarfsdeckendes Angebot erreicht worden?

\section{Entwicklung der Kinderbetreuung für Kleinkinder in Westeuropa}

Die Betreuung von Kleinkindern ist ein Politikfeld, das erst in jüngerer Zeit an Bedeutung gewonnen hat. Außerdem ist es zumeist auf den unteren staatlichen Ebenen angesiedelt. Aus diesen Gründen ist die Datenlage hier außerordentlich prekär: Lange, international vergleichbare Zeitreihen liegen nicht vor. Die von Eurostat auf der Basis der EU-SILC-Erhebung bereitgestellten Kennziffern sind ohne Kenntnis der nationalen Betreuungssysteme kaum zu interpretieren. Um dennoch zu brauchbaren Aussagen über die Entwicklung in den beiden letzten Jahrzehnten zu kommen, kombinieren wir Daten aus unterschiedlichen Quellen miteinander. Dabei werden nationale Zeitreihen zu Betreuungsquoten bzw. Platz-Kind-Relationen auf der Grundlage qualitativer Länderberichte interpretiert und gegebenenfalls mit internationalen Daten abgeglichen.

Die Analyse erfolgt in drei Ländergruppen, in denen die Kinderbetreuung jeweils in ähnlicher Weise organisiert ist: Zunächst gehen wir auf die skandinavischen Wohlfahrtsstaaten ein. Danach behandeln wir eine Ländergruppe, in der die öffentliche Kinderbetreuung auf der Vorschule basiert. Abschließend werden Deutschland und andere Länder untersucht, bei denen die formale Kinderbetreuung traditionell entweder eine Halbtagsbetreuung darstellt oder kaum eine Bedeutung besitzt. 


\subsection{Skandinavien: ausgebaute Transfers und Dienstleistungen}

Eine Gemeinsamkeit der skandinavischen Staaten ist das weitgehend bedarfsdeckende, zumeist ganztägige Angebot an Kleinkindbetreuung. Die Länder in dieser Gruppe unterscheiden sich jedoch im Zusammenspiel von Geld- und Dienstleistungen, was sich in Unterschieden bei den Betreuungsquoten niederschlägt.

Die in Abbildung 1 ausgewiesene Betreuungsquote für die finnischen Kleinkinder liegt weit unterhalb dessen, was von einem skandinavischen Land gemeinhin erwartet wird. Ursache dafür ist das 1985 eingeführte Betreuungsgeld. Dieses ist durch verschiedene Zulagen auf lokaler Ebene weitaus großzügiger als die Betreuungsgeldleistungen in den anderen nordischen Ländern (Ellingsæter 2012, S. 5). Das Betreuungsgeld stand der Einführung eines subjektiven Rechts auf formale Betreuung für Kleinkinder im Jahre 1996 nicht entgegen. Es sorgte vielmehr dafür, dass das öffentliche Betreuungsangebot seit Anfang der 1990er Jahre stets dem Bedarf entsprach (Sutela 2008, S. 2). Die im Vergleich zu den anderen skandinavischen Staaten niedrigen Betreuungsquoten sind also nicht das Ergebnis einer unzureichenden Verfügbarkeit, sondern freier Wahl. Ähnlich verhält es sich bei den Öffnungszeiten: Die Kommunen sind gesetzlich verpflichtet, Kinderbetreuung zu einer Zeit und in einer Form anzubieten, die der Nachfrage entspricht. Wenngleich es in eiligen Fällen oder am Wochenende zu Schwierigkeiten kommen kann, stellen die Öffnungszeiten, welche sich sogar auf die Nacht und das Wochenende erstrecken, aus vergleichender Perspektive kein Problem dar (ebd. S. 7f.).

In Schweden begann der Ausbau der öffentlichen Kinderbetreuung bereits 1973. Die Kommunen sind 1995 per Gesetz verpflichtet worden, Kindern mit studierenden, arbeitenden, arbeitslosen und bedürftigen Eltern innerhalb von drei oder vier Monaten Betreuungsplätze anzubieten (Eydal/Rostgaard 2011, S. 79; NOSOSCO 2010, S. 59f.). Etwa zehn Jahre später waren fast alle Kommunen dazu auch tatsächlich in der Lage. Die Betreuung erfolgt überwiegend ganztags. In der Mehrheit der Kommunen wird die Kinderbetreuung auch abends und am Wochenende angeboten (Nyberg 2008, S. 8; Skolverket 2009, Tabellen 1a und 2). Die lange Anspruchsdauer und die großzügige Lohnersatzquote des Elterngeldes führen jedoch dazu, dass die Betreuungsquoten niedriger sind als etwa in Dänemark. Das 2008 eingeführte Betreuungsgeld in Höhe von etwa $340 €$ hat hingegen kaum eine Bedeutung (Ellingsæter 2012, S. 5, 7f.). Tatsächlich ist es so, dass das Angebot dem Bedarf entspricht (Skolverket 2011, S. 8).

Auch in Norwegen hat in den letzten zwei Jahrzehnten ein rascher Ausbau der öffentlichen Betreuung von Kleinkindern stattgefunden. Wies das Land im Jahre $1990 \mathrm{mit}$ nur $11 \%$ die niedrigste Betreuungsquote unter den skandinavischen Sozialstaaten auf, so war der Wert 2010 mit 55,5\% sogar deutlich überdurchschnittlich. Die zu-
ABB. 1

Kleinkindbetreuung in den nordischen Ländern 1990 - 2010

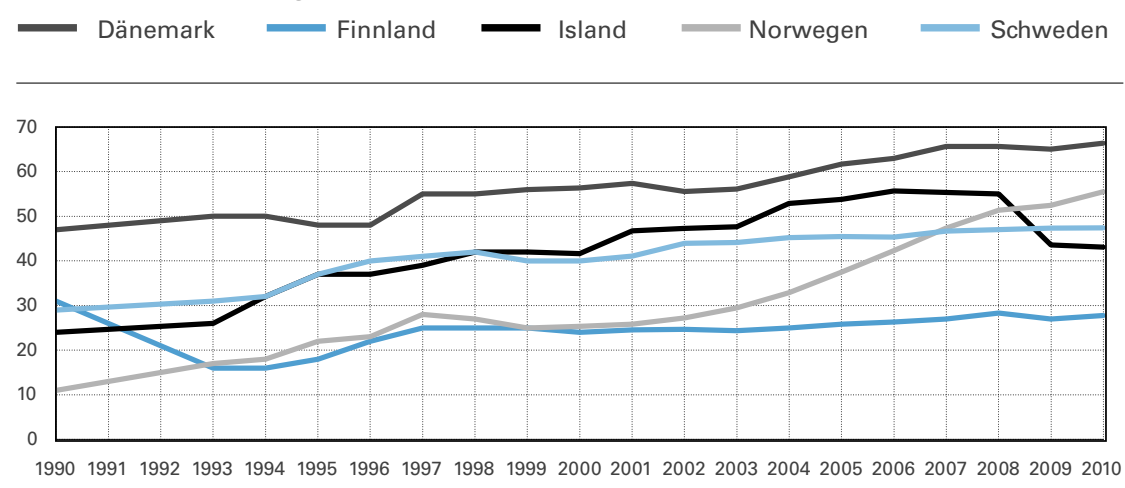

Quelle: Seils (2014); NOSOSCO, verschiedene Jahrgänge.

sätzlichen Plätze wurden zu einem großen Teil in sogenannten „Familienkindergärten“ innerhalb elterlicher Wohnungen geschaffen (Ellingsæter 2008, S. 15), welche in der Praxis Tagesmüttern sehr nahe kamen. Dennoch konnte der rasche Ausbau der öffentlichen Betreuung die Nachfrage lange Zeit nicht befriedigen, weil diese ebenfalls stieg. Auch das im Jahre 1998 eingeführte Betreuungsgeld in Höhe von etwa $400 €$ hat daran nichts geändert. Die Zahl der Empfängerinnen ist mit dem Ausbau der öffentlichen Kinderbetreuung vielmehr gesunken (Ellingsæter 2012, S. 5f.). Das weitaus großzügigere Elterngeld, das 44 Wochen lang an Elternteile ausgezahlt wird, die sich der Betreuung ihres Kindes widmen, war hingegen in der Lage, den Bedarf an formaler Kinderbetreuung im ersten Lebensjahr zu reduzieren. Seit 2005 hat die Mitte-LinksRegierung die Transfers an die Kommunen noch einmal deutlich aufgestockt, um die Zahl der Plätze zu erhöhen. Seit 2009 existiert schließlich eine Verpflichtung für die Gemeinden, allen Eltern für Kinder nach dem vollendeten ersten Lebensjahr einen Betreuungsplatz anbieten zu können.

Die Ausweitung der Betreuung hat auch im Bereich der täglichen Nutzung der Kindergärten stattgefunden, sodass Norwegen heute fast ausschließlich Vollzeitbetreuung kennt. Waren im Jahre 2001 noch 64,3 \% aller Kindergartenkinder mehr als 32 Stunden in der Woche im Kindergarten, so ist der Anteil der Vollzeitplätze bis 2011 auf 93,1 \% gestiegen (Statistics Norway 2012).

Dänemark war 1964 das erste Land, das eine gesetzliche Grundlage für die öffentliche Kinderbetreuung schuf. Heute ist Dänemark unangefochten das Land in Europa mit der höchsten Betreuungsquote in der Altersgruppe unter drei Jahren. Obwohl sich nach nationaler Definition bereits 1990 etwa die Hälfte aller Kinder unter drei Jahren in formaler Betreuung befand, gab es zu dieser Zeit noch lange Wartelisten für Betreuungsplätze. Seit Mitte der 
ABB. 2

\section{Kleinkindbetreuung in Ländern mit Vorschultradition1990 - 2010}
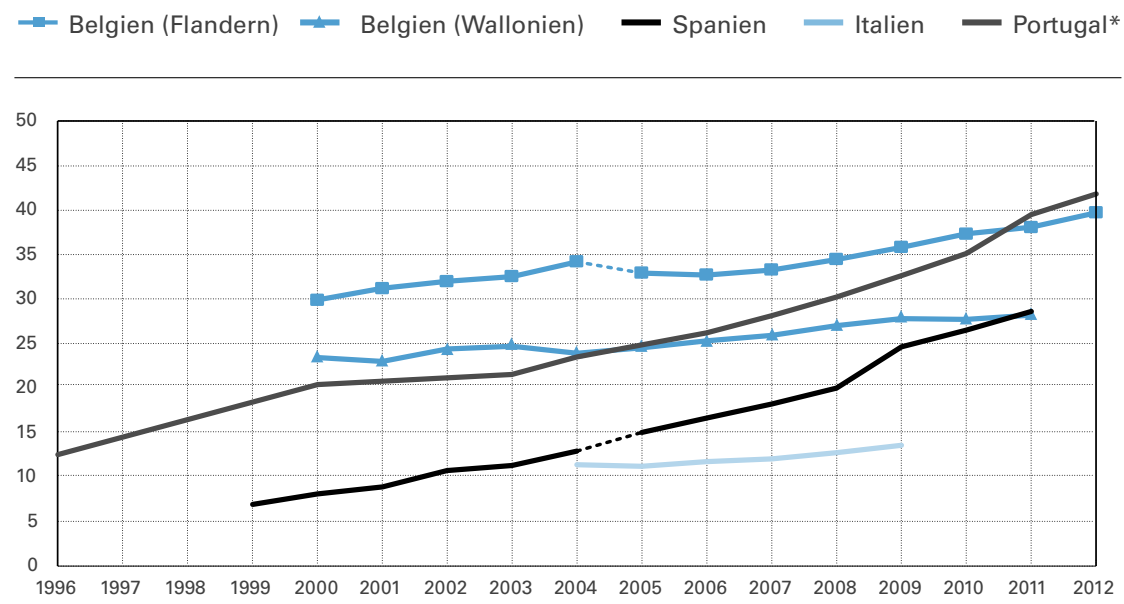

* Die Angaben für Portugal beziehen sich auf Kinder unter vier Jahren.

Quelle: Seils (2014); nationale Behörden. bot zu sorgen, es besteht jedoch keine individuelle Garantie auf einen Betreuungsplatz. Die Betreuungsquote stieg zwischen 1990 und 2008 von 24 auf $55 \%$. Allerdings ist sie danach - mutmaßlich infolge der Finanzkrise - eingebrochen und lag zuletzt bei nur noch $43 \%$.

Die Geldleistungen in Island sind weniger großzügig als in den anderen nordischen Ländern. Das Elterngeld ersetzt nur 39 Wochen lang 75-80\% des letzten Einkommens (NOSOSCO 2011, S. 40ff.). Ein nationales Betreuungsgeld besteht auch nicht. Allerdings haben die wichtigsten Städte im Jahre 2006 ein Betreuungsgeld eingeführt (Eydal/Rostgaard 2011, S. 90), um Lücken im Betreuungsangebot zu schließen, die nach dem Auslaufen des Elterngeldes entstehen können (Mósesdóttir 2008, S. 13). Dieses wird jedoch nur an solche Kinder gezahlt, die auf einer Warteliste für einen Betreuungsplatz stehen. Im Zuge der Finanzkrise wurde das Betreuungsgeld 2011 in Reykjavik jedoch wieder abgeschafft (Eydal/Rostgaard 2011, S. 90ff.). Nationale Daten belegen zudem einen Trend zur Ganztagsbetreuung. Nach den neuesten Zahlen (2012) befinden sich 98 \% der Kleinkinder mehr als fünf Stunden pro Tag in der Vorschule, sodass weiteres Wachstum auch im Bereich der täglichen Nutzungsdauern an Grenzen stößt. ${ }^{1}$

1990er Jahre wurde die Kinderbetreuung weiter ausgebaut. 1996 brachte die sozialdemokratische Regierung unter Paul Nyrup Rasmussen die Idee einer allgemeinen Betreuungsgarantie auf die Tagesordnung, wonach ab 2000 jedes Kind vor dem Eintritt in die Vorschule ein Recht auf einen Betreuungsplatz erhalten sollte. $\mathrm{Zu}$ diesem $\mathrm{Zweck}$ wurden den Gemeinden zusätzliche Finanzmittel zur Verfügung gestellt. Seit 2004 sind die Kommunen tatsächlich verpflichtet, eine ausreichende Betreuung sicherzustellen (NOSOSCO 2010, S. 59; Obinger et al. 2010, S. 102, 107f.). In der Folge stieg die Betreuungsquote in der Abgrenzung von NOSOSCO bis zum Jahre 2008 auf $66 \%$. Sie hat damit ein Niveau erreicht, auf dem weiteres Wachstum in der Altersgruppe über einem Jahr nur noch begrenzt möglich ist, da bereits über $80 \%$ dieser Kinder einen Kindergarten besuchen. Die Betreuungsquote der Kinder unter einem Jahr liegt hingegen mit $15 \%$ deutlich niedriger, was vor allem auf das großzügige Elterngeld zurückzuführen sein dürfte, welches im ersten Lebensjahr des Kindes gezahlt wird. Dieses ersetzt das verlorene Arbeitseinkommen bis zu einer Obergrenze vollständig (NOSOSCO 2009, S. 38f.; Statistics Denmark 2012, S. 134). Außerdem wurden die Kommunen 2002 ermächtigt, ein Betreuungsgeld anzubieten. Tatsächlich sind die Anspruchsvoraussetzungen aber so strikt, dass die Leistungen kaum abgerufen werden (Eydal/Rostgaard 2011, S. 89ff.). Die Betreuung der Kleinkinder findet ganz überwiegend ganztags statt. Jedoch finden sich in der Literatur Hinweise darauf, dass die Öffnungszeiten der Kindertagesstätten nicht immer der Nachfrage der Eltern entsprechen (Emerek 2008, S. 17).

Ausgehend von einem hohen Niveau hat sich die Kinderbetreuung in Island seit 1990 rasant entwickelt, sodass fast Vollversorgung herrschte. Zwar sind die Kommunen hier verpflichtet, für ein bedarfsgerechtes Betreuungsange-

\subsection{Kleinkindbetreuung vor der Vorschule}

Frankreich wird oft als das Ursprungsland der formalen Kinderbetreuung angesehen, da die Vorschulen bereits 1881 in das öffentliche Bildungssystem integriert wurden (Lechevalier 2014; Oberhuemer et al. 2010, S. 147f.). Mit Belgien, Portugal, Spanien und Italien gibt es jedoch eine ganze Reihe von Ländern, die ein eigenes mehr oder minder ausgebautes Vorschulsystem für Kinder ab 2,5 bzw. drei Jahren etabliert haben. Seit die Nachfrage nach Kleinkindbetreuung steigt, stehen diese Länder vor der Frage, ob und wie sie die Betreuung von Kindern unter drei Jahren organisieren wollen. Über Ländergrenzen hinweg zeigt sich, dass das Betreuungsangebot in dieser Altersgruppe bislang deutlich weniger ausgebaut (Abbildung 2) und stärker institutionell differenziert ist als jenes für die Kinder im Vorschulalter.

In Frankreich haben in diesem Zusammenhang die Tagesmütter eine große Bedeutung erlangt. Die Ursache dieses Booms liegt in einer 1980 eingeführten finanziellen Unterstützung für Eltern, die ihre unter dreijährigen Kinder in der Wohnung von einer zertifizierten Tagesmutter (assistante maternelle agréée) betreuen lassen. Diese Leistung wurde 1990 ausgebaut und deckt nun die Sozialabgaben der Tagesmütter ab (Leitner 2009, S. 378). Außerdem wird eine Steuervergünstigung gewährt (Lechevalier 2014). In den folgenden beiden Jahrzehnten stieg der Anteil der Tages-

1 Berechnungen der Autoren auf Basis von Statistics Iceland, http://www.statice.is/Statistics/Education/ Pre-primary-institutions. 
mütter-Plätze schnell an. Gab es 1990 nur für 5,9\% der Kinder unter drei Jahren einen solchen Platz, waren es im Jahre 2000 schon über 27,3\%. Nach den letzten verfügbaren Zahlen waren es 2010 35,2 \% (Observatoire National De La Petite Enfance 2012, S. 12). Daneben wird bereits seit 1986 die Betreuung durch Hausangestellte bzw. Kindermädchen durch eine Beihilfe (allocation de garde d'enfant à domicile) vom französischen Staat gefördert. Diese deckt einen Teil der Sozialabgaben ab. Außerdem können die Lohnkosten in einem bestimmten Rahmen von der Einkommensteuer abgesetzt werden, was vor allem für Haushalte mit hohem Einkommen interessant ist. Heute werden noch etwa 1,9\% der Kinder in der Altersgruppe von angestellten Kindermädchen (salarié à domicile) betreut.

Die in den meisten anderen europäischen Ländern dominierende Betreuung in Einrichtungen ist in Frankreich weit weniger bedeutend und hat kaum zugenommen. Die Platz-Kind-Relation in diesem Bereich nahm zwischen 2000 und 2010 nur um etwa zwei Prozentpunkte zu und betrug zuletzt 15,1\%. ${ }^{2}$ Die Bedeutung der Vorschulen (école maternelle) für die Betreuung der Kinder unter drei Jahren nahm in den letzten Jahren sogar ab. Während die nationale Statistik für 2010 eine Platz-Kind-Relation von 49,9\% für Kleinkinder ausweist (Observatoire National De La Petite Enfance 2012, S. 22), ${ }^{3}$ kommt Eurostat für dasselbe Jahr auf eine Betreuungsquote von $43 \%$. Trotz der vergleichsweise hohen Quoten kommen nationale Beobachter zu dem Ergebnis, dass das Angebot in dieser Altersgruppe nicht bedarfsdeckend ist (Lechevalier 2014; Oberhuemer et al. 2010, S. 151).

Im Nachbarland Belgien liegt die Betreuung von Kindern unter 2,5 Jahren in der Kompetenz der flämischen bzw. wallonischen Gemeinschaften. Im wallonischen Teil Belgiens wird die Kinderbetreuung vom Office National de la Naissance et de l'Enfance (ONE) akkreditiert und subventioniert. Seit 2000 hat die Zahl der Betreuungsplätze pro 100 Kinder von 23,4 auf 28,2 (2011) zugenommen. ${ }^{4}$ Dies mag auch auf den Plan Coignant von 2003 zurückzuführen sein, der darauf ausgerichtet war, die Zielsetzungen von Barcelona einzuhalten. Berechnungen von ONE legen nahe, dass diese in international vergleichbarer Altersabgrenzung mit 44,5\% bei Weitem übertroffen wurden. Die subventionierten Einrichtungen sind an 220 Tagen im Jahr zehn Stunden lang geöffnet (Meulders/O'Dorchai 2008, S. 15).

Im flämischen Teil wird die Kinderbetreuung durch eine Anstalt namens Kind en Gezin beaufsichtigt und subventioniert, die dem flämischen Sozialministerium verantwortlich ist. Die Betreuung der Kinder unter drei Jahren ist auch in Flandern seit dem Jahre 2000 ausgebaut worden. Im Jahre 2012 waren für 22,6 bzw. 39,7 von 100 Kindern (subventionierte) Betreuungsplätze vorhanden. Da diese im Falle von Halbtagsbetreuung von mehreren Kindern genutzt werden können, liegt die Betreuungsquote in der Altersgruppe deutlich höher. Außerdem ist zu berücksichtigen, dass viele 2,5-Jährige in die Vorschule gehen, sodass etwa sechs von zehn Kleinkindern regelmäßig formal betreut werden. Dennoch herrscht eine große Knappheit an formaler Betreuung (Oberhuemer et al. 2010, S. 37; Kind \& Gezin 2012, S. 36, 39). Die geförderten Betreuungsstätten sind ebenfalls an 220 Tagen im Jahr etwa elf Stunden pro Tag geöffnet (Kind \& Gezin 2011, S. 5).

Die Ursprünge der portugiesischen Vorschulen liegen im 19. Jahrhundert. Allerdings wurden diese während der Diktatur abgeschafft und erst kurz vor der Revolution in den frühen 1970er Jahren wieder eingeführt. Gegen Ende der 1960er Jahre wurden auch die ersten Kindergärten für Kleinkinder eingeführt (Ministry of Education 2001, S. 15ff.). Bis zur Mitte der 1990er Jahre gab es jedoch nur für 12,5\% der Kinder unter vier Jahren einen Betreuungsplatz (Formosinho/Formosinho 2008, S. 16). Die vorhandenen Institutionen lassen sich grob in registrierte Tagesmütter (amas) und Tageseinrichtungen (creches) unterschiedlicher Art und Größe einteilen (ebd. S. 5f.). Ein erster Schritt zur Ausdehnung der formalen Betreuung von Kindern zwischen vier Monaten und drei Jahren war das PAPI-Programm aus dem Jahre 2000. Einer nationalen Beobachterin zufolge hatte das Programm jedoch bestenfalls einen eng begrenzten Erfolg (Ferreira 2008, S. 6). Im Jahre 2006 beschloss die Regierung ein ehrgeiziges Programm, welches den Aufbau neuer Tageseinrichtungen vorsah, um die Platz-Kind-Relation bis 2010 auf $35 \%$ anzuheben (ebd. S. 6; Formosinho/Formosinho 2008, S. 13). Der größte Teil der zusätzlichen Betreuungsplätze ist auf den Ausbau der Kindergärten zurückzuführen, während die Zahl der Plätze bei den Tagesmüttern seit 2005 sogar abnimmt. Schließlich trugen sinkende Geburtenzahlen ebenfalls dazu bei, dass die Platz-Kind-Relation zwischen 2006 und 2010 von 26,2 auf 35,2\% anstieg. Nach aktuellen Zahlen liegt die Platz-Kind-Relation bei 41,8 \% (Gabinete De Estratégia E Planeamento verschiedene Jahrgänge). Außerdem unternahm die Regierung Schritte, um die Öffnungszeiten der Kindergärten an die Arbeitszeiten der Eltern anzunähern. Trotz des beeindruckenden Wachstums der Kleinkindbetreuung wird die öffentlich geförderte frühkindliche Bildung insgesamt von nationalen Expertinnen noch stets als unzureichend angesehen (Pinto et al. 2013, S. 94).

Spanien blickt ebenfalls auf eine Vorschultradition, die in das 19. Jahrhundert zurückreicht. Allerdings wurde die Expansion der frühkindlichen Bildung wie in Portugal bis Mitte der 1970er Jahre durch eine Diktatur beendet (Oberhuemer et al. 2010, S. 415). Noch im Jahre 1999 waren

2 Die Differenzen sind auf Basis der Série enquête PMI und Bevölkerungsdaten von Eurostat berechnet worden. Die Platz-Kind Relation stützt sich auf die Angabe der Série CNAF-DREES.

3 Bei dieser Angabe ist jedoch zu berücksichtigen, dass Doppelzählungen nicht ausgeschlossen werden können.

4 Die Zahlen beziehen sich auf Kinder unter 2,5 Jahren. 
nur 6,9 \% aller Kinder unter drei Jahren in formaler Betreuung. Allerdings stieg die Betreuungsquote in den folgenden Jahren kontinuierlich an. Im Jahre 2006 schloss sich Spanien den bildungspolitischen Zielen der Europäischen Union an. Dazu sollte die öffentliche Betreuung von Kleinkindern ausgedehnt werden. In der Tat erhöhte sich in der Folge die Betreuungsquote bis auf 28,6 \% im Jahre 2011. Folgt man der Literatur, dann reichte das öffentliche Angebot auch in den letzten Jahren nicht aus, um den Bedarf zu decken. Zugleich waren private Alternativen oft unerschwinglich (Gago 2008, S. 8). Zudem steht die Qualität der Betreuung gerade bei privaten Einrichtungen infrage (Oberhuemer et al. 2010, S. 417f.).

In Italien wird die universelle Vorschule (scuola infanzia) seit den frühen 1970er Jahren durch eine öffentliche Kleinkindbetreuung ergänzt. Auf Druck der Gewerkschaften und der Frauenbewegung wurde 1971 die gesetzliche Grundlage für kommunale Betreuungseinrichtungen (asili nido) geschaffen. Diese bieten eine Ganztagsbetreuung von neun Stunden zwischen 7.30 und 18.00 Uhr an, wobei im Juli nur ein verringertes Angebot vorhanden ist (Musatti/Picchio 2010, S. 143f.; Simonazzi 2008, S. 7). Die meisten dieser Betreuungseinrichtungen werden von den Kommunen selbst betrieben oder subventioniert. Die Elternbeiträge decken im Durchschnitt gut 20 \% der Kosten ab. Allerdings blieb das Angebot im Landesdurchschnitt bescheiden und fiel regional sehr unterschiedlich aus. Um dem wachsenden Bedarf zu begegnen, wurde 2007 ein Pilot-Programm zum Ausbau der Kleinkindbetreuung aufgelegt. Dazu wurde das Eintrittsalter der Vorschule in zahlreichen Kommunen auf zwei Jahre gesenkt und man schuf besondere Klassen für Kinder zwischen zwei und drei Jahren. Diese sind allerdings im Unterschied zur Vorschule für die Eltern kostenpflichtig (Oberhuemer et al. 2010, S. 244). Außerdem wurde mit den

ABB. 3

\section{Kleinkindbetreuung in Mitteleuropa und Großbritannien 1990 - 2013}

$\begin{array}{lll}- \text { Österreich } & \text { Deutschland } & \text { Niederlande } \\ - \text { - Großbritannien (Schottland) } & - \text { Großbritannien (Wales) }\end{array}$

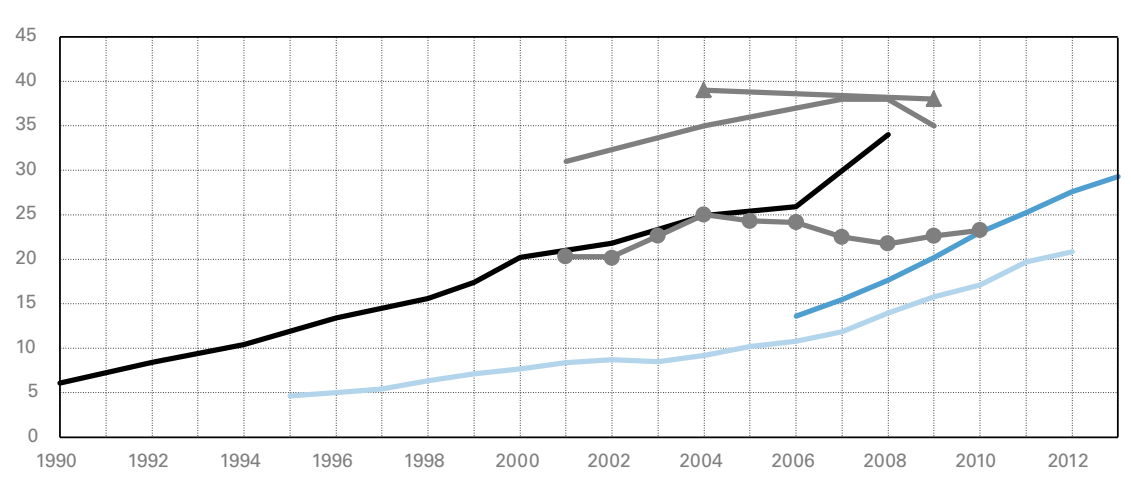

* Die Angaben für die Niederlande beziehen sich auf Kinder unter vier Jahren.

5 Den Zahlen von Eurostat zufolge gehören die Niederlande heute zu den Ländern mit den höchsten Betreuungsquoten in der Altersgruppe unter drei Jahren.

spazio bambini eine Halbtagsbetreuung für Kinder zwischen 18 und 36 Monaten geschaffen. Den Angaben des italienischen statistischen Amtes (ISTAT) zufolge haben diese zusätzlichen Anstrengungen zwischen 2004 und 2009 jedoch nur zu einem geringen Anstieg der Betreuungsquote der Kleinkinder von 11,4 auf 13,6\% geführt. Angesichts des unzureichenden öffentlichen Betreuungsangebotes und der geringen Bedeutung von Tagesmüttern und Hausangestellten kommt den Großeltern daher bei der Betreuung von Kleinkindern eine wachsende Rolle zu (Sarti 2010, S. 795ff.).

\subsection{Kleinkindbetreuung im Aufbruch?}

Es verbleibt eine ganze Reihe von Ländern, deren Gemeinsamkeit in Hinblick auf die formale Betreuung von Kleinkindern nur darin besteht, dass diese sich nur auf wenige Kinder erstreckt oder nur halbtags angeboten wird. Diese heterogene Gruppe umfasst zum einen die Länder der KindergartenTradition (Deutschland, Österreich, Schweiz), und zum anderen die Länder der Armuts-Tradition (Großbritannien, Irland). Die Niederlande und Luxemburg lassen sich keiner der beiden Richtungen zuordnen und werden daher gleich zu Beginn abgehandelt. Insgesamt stellt sich die Frage, inwiefern die formale Betreuung der Kinder unter drei Jahren auch in dieser Gruppe ausgedehnt wird (vgl. Abbildung 3).

In den Niederlanden hat der Ausbau der Kleinkindbetreuung inzwischen zu einem beachtlichen Leistungsniveau geführt. Die nationale Betreuungsquote hat sich in den letzten zwanzig Jahren enorm entwickelt. Sie stieg von 6,1\% der Kinder unter vier Jahren (1990) auf 34 \% im Jahre 2010 an (Merens et al. 2011). ${ }^{5}$ Allerdings ist hier in Rechnung zu stellen, dass die Eltern zumeist nur eine Halbtagsbetreuung in Anspruch nehmen (Plantenga/Remery 2008).

Die Initialzündung für diese rasante Entwicklung stellte ein Subventionsprogramm der niederländischen Regierung von 1990 dar. Dies führte bis zum Ende der 1990er Jahre zu einem Flickenteppich von subventionierten, arbeitgeberfinanzierten und privaten Einrichtungen, was zu einer ungleichen Versorgung und erheblichen Unterschieden bei den Kosten führte, die bei den Eltern anfallen. Das Kinderbetreuungsgesetz vom Januar 2005 reformierte die formale Betreuung dahin gehend, dass nicht mehr das Angebot bereitgestellt, sondern die Nachfrage durch den Staat und den Arbeitgeber subventioniert wird. Gleichzeitig wurde der Kostenanteil der Eltern auf das in Skandinavien vorherrschende Niveau gesenkt (ebd.). Allerdings hat die Regierung den Betreuungszuschuss im Jahre 2009 erheblich gekürzt, was die Kosten für Familien in die Höhe getrieben hat. In der Folge haben manche Familien ihre Kinder wieder informell betreuen lassen (Merens et al. 2012, S. 94f., 188). In den Jahren 2012 und 2013 wurde der Elternbeitrag jeweils 
weiter erhöht und eine Obergrenze für die Zahl der subventionierten Stunden eingeführt. ${ }^{6}$

In Luxemburg ist die öffentlich finanzierte Betreuungsinfrastruktur für Kinder bis zu zwei Jahren deutlich ausgebaut worden (Plasman 2008, S. 6). Dennoch bestand gerade für diese Altersgruppe in der ersten Hälfte des letzten Jahrzehnts noch eine lange Warteliste. Im Jahr 2003 wurden die ersten Tagesmütter zugelassen. ${ }^{7}$ Die Zahl der Plätze bei diesen Tagesmüttern lag im ersten Jahr bei 90. Im Jahre 2008 waren es schon 1296 und Ende 2011 wurden schließlich 2570 Plätze angeboten (Ministère de la Famille et l'intégration 2012, S. 135). Außerdem wurde die Zahl der Plätze in den Betreuungseinrichtungen weiter ausgebaut. Dennoch verkürzte sich die Warteliste auf einen Kindergartenplatz zwischen 2003 und 2008 nur von 5,5 auf 4,2\% aller Kinder in der Altersgruppe unter drei Jahren. ${ }^{8}$ In Bezug auf die tägliche Nutzungsdauer weisen die nationalen Zahlen darauf hin, dass sich im Durchschnitt aller Altersgruppen etwa drei Viertel der Kinder in Ganztagsbetreuung befinden.

Die öffentliche Förderung der Kleinkindbetreuung in Großbritannien und Irland konzentriert sich stark auf Kinder sozial schwacher Eltern. In Großbritannien wurde dazu im Jahre 1998 das Sure-Start-Programm aus der Taufe gehoben. Es sollte die Dienstleistungen für die Gesundheit bzw. Bildung der Kinder und die finanzielle Unterstützung besser koordinieren (Roberts 2000). Nach den letzten verfügbaren Daten befanden sich in England $35 \%$ der Kinder unter drei Jahren in formaler Betreuung (Smith et al. 2010, S. 31, Tabelle 2.3). Wie Abbildung 3 zeigt, liegen die Betreuungsquoten in Schottland deutlich niedriger und in Wales knapp unter $40 \%$. Allerdings ist zu berücksichtigen, dass es sich dabei keinesfalls ausschließlich um öffentlich subventionierte Betreuung handelt. Nationalen Angaben zufolge wurden die englischen Kindergartenkinder in der Altersgruppe unter drei Jahren 2009 durchschnittlich 20 Stunden in der Woche formal betreut (Smith et al. 2010, S. 39, Tabelle 2.6), was auf eine große Verbreitung der Teilzeitbetreuung schließen lässt. Der vorhandene Umfang an formaler Kinderbetreuung wird von den Eltern als unzureichend eingeschätzt. Dies zeigt sich auch daran, dass $8 \%$ der arbeitenden Eltern in England angeben, auf einer Warteliste für einen Kindergartenplatz zu stehen. Zudem nutzen $16 \%$ der werktätigen Eltern aufgrund der Kosten keinen Kindergarten für ihr Kind. (Smith et al. 2010, S. 101, Tabelle 6.10).

In Irland gibt es trotz einiger Anläufe zum Aufbau einer öffentlich finanzierten Kinderbetreuung seit den 1990er Jahren nur wenige öffentlich geförderte Zentren in sozialen Brennpunkten. Diese auf Armutsbekämpfung konzentrierte Kleinkindbetreuung besteht aus dem bereits 1969 ins Leben gerufenen Rutland-Street-Project und dem Early-Start-Programme (Hayes 2010, S. 72). Letzteres unterhält laut Angaben des irischen Departments of Education and Skills 40 Zentren mit 1650 Plätzen. Beide Programme beschäftigen sich außerdem nur mit kleinen Kindern nach ihrem dritten Geburtstag.

Die verfügbaren Statistiken beruhen auf Haushaltsbefragungen, die jedoch nicht nach dem Alter des Kindes aufge- schlüsselt sind. Diese stützen jedoch die Vermutung, dass die formale Betreuung für Kleinkinder in Irland ganz überwiegend aus privat finanzierter Kinderbetreuung, Spielgruppen, Tagesmüttern und Au Pair besteht (Barry 2008, S. 2ff.; Central Statistics Office 2009, S. 4). Angesichts der Tatsache, dass $60 \%$ der Eltern meinen, dass sie in ihrer Umgebung keinen Zugang zu qualitativ hochwertiger und bezahlbarer Betreuung haben (Central Statistics Office 2009, S. 21), kann von einer bedarfsdeckenden Versorgung keine Rede sein (Hayes 2010, S. 76). Die Folge ist, dass berufstätige Eltern für die Kinderbetreuung sehr häufig auf die Großeltern zurückgreifen müssen.

Die deutschsprachigen Länder weisen eine gemeinsame Kindergarten-Tradition auf, die sich von der Vorschule durch die Halbtagsbetreuung und die Betonung des kreativen Spiels gegenüber Bildungsaspekten unterscheidet. In der Altersgruppe über drei Jahren gehörte Deutschland 1996 zu den Ländern, die bereits früh eine Betreuungsgarantie eingeführt haben. Die formale Betreuung von Kindern unter drei Jahren kam in Deutschland hingegen erst Mitte des vergangenen Jahrzehnts auf die politische Tagesordnung. Die erste greifbare Folge war das Tagesbetreuungsausbaugesetz, welches 2005 in Kraft trat. Darin wurde ein Ausbau der öffentlichen Betreuung festgeschrieben. Bis zum Oktober 2010 sollten danach 230.000 neue Plätze geschaffen werden. Die aktuelle Diskussion wird jedoch von den Ergebnissen des sogenannten Krippengipfels des Jahres 2007 bestimmt. Noch bevor die Zielsetzungen des Tagesbetreuungsausbaugesetzes erreicht waren, wurde beschlossen, den Rechtsanspruch auf einen Teilzeitplatz auf alle Kinder nach Vollendung des ersten Lebensjahres auszudehnen. Im Kinderförderungsgesetz von 2008 wurde ein Rechtsanspruch vorgesehen, der seit August 2013 in Kraft ist. Diese Betreuungsgarantie bezieht sich jedoch im Unterschied zu denen in den skandinavischen Ländern nur auf eine Halbtagsbetreuung. Die Gesetzgebung hat sich in einem rasanten Anstieg der Betreuungsquote niedergeschlagen. Waren 2006 erst 13,6\% der Kinder unter drei Jahren in einem Kindergarten oder bei einer Tagesmutter, so waren es im März 2013 schon 29,3\%. Gegenüber dem von der Bundesregierung ermittelten Bedarf von etwa 39 \% (BMFSFJ 2012, S. 1) besteht damit eine Lücke von zehn Prozentpunkten. Trotz des Anstieges der Betreuungsquote kann somit die vorhandene Nachfrage insbesondere in den Ballungsgebieten des

6 Diese Angaben stützen sich auf das Centraal Bureau voor de Statistiek, http://www.cbs.nl/nl-NL/menu/methoden/toelich tingen/alfabet/n/nieuwe-regels-kinderopvangtoeslag.htm.

7 In Luxemburg sind genaue Angaben zu speziellen Altersgruppen kaum möglich, da die Kantone unterschiedliche Abgrenzungen verwenden. Dies führt dazu, dass die einzelnen Angaben auf nationaler Ebene nicht mehr zusammengefasst werden können.

8 Berechnungen der Autoren auf der Basis der Jahresberichte des Familienministeriums (Ministère de la Famille et I'intégration 2004, S. 79; 2009, S. 68) und Bevölkerungsangaben von Eurostat. 
Westens derzeit nicht befriedigt werden. Die Betreuungszeiten stellen hingegen gegenwärtig kein großes Problem dar.

Österreich hat in der zweiten Hälfte der 1990er Jahre ein Sonderprogramm aufgelegt, das den Mangel an Betreuungsplätzen für Kinder zwischen drei und sechs Jahren reduzierte. Die zweite Stufe des Programms, bei der es um den Ausbau der Kinderbetreuung für unter Dreijährige und Schulkinder ging, wurde nach dem Regierungswechsel des Jahres 2000 auf Eis gelegt. Stattdessen wurde mit der Einführung des Kinderbetreuungsgeldes ein zentrales Wahlversprechen der schwarzblauen Koalition umgesetzt. Die Kinderbetreuung wurde trotz des unbefriedigten Bedarfes von dieser Regierung nicht weiter vorangetrieben (Obinger/Tálos 2006, S. 173ff.). Allerdings bewirkte das Kinderbetreuungsgeld allenfalls 2003 einen kleinen Rückgang der Betreuungsquote der Kinder unter drei Jahren (Baierl/Kaindl 2011, S. 37). Statistik Austria (2013) zufolge ist der Anteil der Kleinkinder, die eine Betreuungseinrichtung besuchen, von 1995 bis 2012 praktisch ungebrochen von 4,6 auf 20,8\% gestiegen. Außerdem werden gut $2 \%$ aller Kinder in der Altersgruppe von einer Tagesmutter betreut (Baierl/Kaindl 2011, S. 37). Die Öffnungszeiten und die Verweildauer der Kleinkinder sind dabei überraschend lang: Etwa 58 \% der Kinder in den Krippen besuchten die Einrichtung ganztags und über dreiviertel nahmen 2012 in der Betreuungseinrichtung ihr Mittagessen ein.

In der Schweiz hängt nur der deutschsprachige Landesteil der Kindergartentradition an, während sich in den übrigen Gebieten der italienische bzw. französische Einfluss bemerkbar macht. Das Angebot an öffentlich finanzierter Kinderbetreuung in der Altersgruppe unter drei Jahren ist jedoch überall außerordentlich beschränkt. Aufgrund des Mangels an Betreuungseinrichtungen wurde 2003 auf Bundesebene ein auf acht Jahre befristetes Subventionsprogramm aufgelegt, das neue Plätze für Kinder im Alter von zwei Monaten bis zum (Vor)schuleintritt geschaffen hat. 2007 wurde zusätzlich für drei Jahre ein Gutscheinsystem eingeführt, mit dem die Eltern formale Kinderbetreuung kaufen konnten. Insgesamt ist die formale Betreuung von Kleinkindern in der Schweiz außerordentlich schwach entwickelt. Die Schweizerische Konferenz der kantonalen Erziehungsdirektoren (EDK 2007, S. 53) kommt gar zu dem Schluss, dass die außerhäusliche Betreuung von Kindern unter drei Jahren in der Schweiz hauptsächlich von Verwandten und Freunden geleistet wird.

\section{Vergleichende Auswertung}

Die formale Betreuung von Kleinkindern in Westeuropa hat sehr unterschiedliche Wurzeln. Es lassen sich drei Gruppen unterscheiden: In den skandinavischen Ländern wird die Betreuung von Kleinkindern seit Langem und in erheblichem Umfang durch eine Mischung öffentlicher Geld- und Dienstleistungen beeinflusst. Obschon in Schweden, Dänemark, und Finnland eine Betreuungsgarantie besteht, variieren die Be- treuungsquoten in der Gruppe beachtlich, was auf die unterschiedliche Gestaltung der Eltern- und Betreuungsgelder zurückzuführen ist. Dennoch haben die nordischen Länder ein weitgehend bedarfsdeckendes Angebot erreicht.

Die Länder mit Vorschultradition wie z.B. Frankreich, Belgien und Italien stehen hingegen vor der Frage, wie sie die formale Betreuung der Kinder organisieren sollen, die das Vorschulalter noch nicht erreicht haben. Die politischen Antworten fallen in den einzelnen Ländern durchaus unterschiedlich aus. Während in der Altersgruppe über drei Jahren fast alle Kinder die Vorschule besuchen, ist das Betreuungsangebot für Kleinkinder breit gefächert. Neben Einrichtungen unterschiedlicher Größe spielen Tagesmütter eine beachtliche Rolle. Das Gesamtvolumen ist jedoch geringer als bei den älteren Kindern. Selbst in Frankreich ist es jedoch bis heute nicht gelungen, ein bedarfsdeckendes Angebot an formaler Betreuung für diese Altersgruppe bereitzustellen.

Deutschland gehört schließlich zu einer dritten Gruppe, die rein negativ durch ein geringes Volumen an formaler Betreuung bestimmt ist. Positiv lässt sich die Bundesrepublik ferner den Ländern der Kindergarten-Tradition zuordnen, die die Rolle des Spielens gegenüber Bildungsaspekten betont. Innerhalb dieser Gruppe zeichnet sich die Bundesrepublik seit August 2013 durch eine Betreuungsgarantie für Kinder nach Vollendung des ersten Lebensjahres aus. Im Gegensatz zu den skandinavischen Ländern handelt es sich jedoch nur um einen Anspruch auf eine Halbtagsbetreuung. Außerdem ist die Betreuungsgarantie derzeit nicht flächendeckend umsetzbar. Auch Österreich kann der Kindergarten-Tradition zugeordnet werden. Allerdings ist die öffentliche Betreuung von Kleinkindern noch deutlich schwächer ausgebaut als in Deutschland. Auch der deutschsprachige Teil der Schweiz ist dieser Tradition zuzuordnen, wobei für die Altersgruppe unter drei Jahren kaum öffentliche Förderung besteht.

Großbritannien und Irland können hingegen einer Armuts-Tradition zugeordnet werden. Insofern eine öffentlich subventionierte Kinderbetreuung besteht, zielt sie in diesen Ländern stets auf den Ausgleich sozialer Benachteiligung ab. Während in England, Wales und Schottland immerhin zwischen 20 und $40 \%$ der Kleinkinder formal betreut werden, gibt es für Irland nicht einmal brauchbare nationale Statistiken.

In den Niederlanden treten Kinder nach Vollendung des vierten Lebensjahres in die Schule ein, obschon die Schulpflicht erst ein Jahr später beginnt. Unterhalb dieser Altersgrenze besteht eine vergleichsweise gut ausgebaute öffentlich subventionierte Kinderbetreuung, die aber nur halbtags in Anspruch genommen wird.

Jenseits dieser Unterschiede lässt sich in fast allen Ländern ein Ausbau der öffentlichen Betreuung von Kleinkindern beobachten. Dies zeigt sich sowohl an den wachsenden Betreuungsquoten als auch am steigenden Anteil der Ganztagsbetreuung. Vorreiter sind diesbezüglich die nordischen Länder. Allerdings hinkte das Angebot an Kinderbetreuung auch in diesen Ländern lange der Nachfrage hinterher. Ähnlich wie in der Bundesrepublik heute konnten die eingeführten Betreuungsgarantien in vielen Kommunen nicht umgesetzt wer- 
den. Stattdessen wurden Transferleistungen wie das Betreuungsgeld in Anspruch genommen.

Nach und nach folgten Länder wie die Niederlande, Spanien, Portugal, Belgien, Frankreich und schließlich auch Deutschland und Österreich dem Trend zum Ausbau der öffentlichen Betreuung von Kleinkindern. In Irland und der Schweiz hat hingegen kaum ein Ausbau öffentlicher Kinderbetreuung stattgefunden. Außerdem hat die jüngste Finanzkrise dazu geführt, dass die öffentliche Betreuung von Kleinkindern in Island massiv zurückgegangen ist und in den Niederlanden die Subventionen empfindlich gekürzt worden sind.

\section{LITERATUR}

Baierl, A./Kaindl, M. (2011): Kinderbetreuung in Österreich: Österreichisches Institut für Familienforschung, Wien

Barry, U. (2008): The Provision of Childcare Services in Ireland, external report commissioned by and presented to the EU Directorate-General Employment and Social Affairs, Brüssel

Bundesministerium für Familie, Senioren, Frauen und Jugend (BMFSFJ): (2012): Dritter Zwischenbericht zur Evaluation des Kinderförderungsgesetzes, Berlin Central Statistics Office (2009): Quarterly National Household Survey, http:// www.cso.ie/en/media/csoie/releasespublications/documents/ labourmarket/2007/childcareq42007.pdf

Ellingsæter, A. L. (2008): The Provision of Childcare Services in Norway, external report Commissioned by and Presented to the EU Directorate-General Employment and Social Affairs, Brüssel

Ellingsæter, A. L. (2012): Betreuungsgeld. Erfahrungen aus Finnland, Norwegen und Schweden: Friedrich-Ebert-Stiftung, Berlin

Emerek, R. (2008): The Provision of Childcare Services in Denmark, External Report Commissioned by and Presented to the EU Directorate-General Employment and Social Affairs, Brüssel

Eydal, B. G./Rostgaard, T. (2011): Day-care Schemes and Cash-for-Care at Home, in: Nordic Council of Ministers (Hrsg.): Parental Leave, Child care and Gender Equality in the Nordic Countries: Norden, S. 65-107

Ferreira, V. (2008): The Provision of Childcare Services in Portugal, External report commissioned by and presented to the EU Directorate-General Employment and Social Affairs, Brüssel

Formosinho, J./Formosinho, J. (2008): System of early education/care and professionalisation in Portugal: Staatsinstitut für Frühpädagogik (IFP), München

Gabinete de Estratégia e Planeamento (verschiedene Jahrgänge): Carta Social. Folha Informativa, http://www.cartasocial.pt/

Gago, E. G. (2008): The Provision of Childcare Services in Spain, External report commissioned by and presented to the EU Directorate-General Employment and Social Affairs, Brüssel

Hayes, N. (2010): Childcare? Early Childhood Education and Care? Towards an Integrated Early Years Policy for Young Children in Ireland, in: Early Years 30 (1), S. 67-78

Kind \& Gezin (verschiedene Jahrgänge): Jaarverslag Kinderopvang, Kind \& Gezin, Brüssel

Lechevalier, A. (2014): Auf dem Weg zur Dualisierung? Die französische Sozialversicherung im Wandel, in: Seils, E./Bogedan, C./Leiber, S. (Hrsg.): Sozialversicherung: Wandel, Wirkung, Weiterentwicklung, Wiesbaden (im Erscheinen)

Leitner, S. (2009): Von den Nachbarn lernen? Care-Regime in Deutschland,

Österreich und Frankreich, in: WSI Mitteilungen 62 (7), S. 376-382, http://www. boeckler.de/wsimit_2009_07_leitner.pdf

Merens, A./Hartgers, M./Van Den Brakel, M. (2012): Emancipatiemonitor 2012: Sociaal en Cultureel Planbureau, Den Haag

Merens, A./Van Den Brakel, M./Hartgers, M./Hermans, B. (2011): Emancipatiemonitor 2010, Sociaal en Cultureel Planbureau, Den Haag

Meulders, D./O'Dorchai, S. (2008): Childcare in Belgium, Dulbea Working Paper 08-08, Brüssel

Ministère de la famille et l'intégration (verschiedene Jahrgänge): Rapport d'Activité, Luxembourg

Ministry of Education (2001): Early Childhood Education and Care Policy in Portugal: OECD

Mósesdóttir, L. (2008): The Provision of Childcare Services in Iceland, External report commissioned by and presented to the EU Directorate-General Employment and Social Affairs, Brüssel
Musatti, T./Picchio, M. (2010): Early Education in Italy: Research and Practice, in: International Journal of Early Childhood 42 (2), S. 141-153

Nordic Social-Statistical Committee (NOSOSCO) (verschiedene Jahrgänge): Social Protection in the Nordic Countries, Kopenhagen

Nyberg, A. (2008): The Provision of Childcare Services in Sweden, external report commissioned by and presented to the EU Directorate-General Employment and Social Affairs, Brüssel

Oberhuemer, P./Schreyer, I./Neuman, M. J. (2010): Professionals in Early Childhood Education and Care Systems, Opladen/Ridgebrook

Obinger, H./Starke, P./Moser, J./Bogedan, C./Gindulis, E./Leibfried, S. (2010): Transformations of the Welfare State. Small States, Big Lessons, New York Obinger, H./Tálos, E. (2006): Sozialstaat Österreich zwischen Kontinuität und Umbau: Eine Bilanz der ÖVP/FPÖ/BZÖ-Koalition, Wiesbaden Observatoire National De La Petite Enfance (2012): L'accueil du jeune enfant en 2011: Caisse Nationale d'Allocations Familiales, Paris

Pinto, A. I./Pessanha, M./Aguiar, C. (2013): Effects of home environment and center-based child care quality on children's language, communication, and literacy outcomes, in: Early Childhood Research Quarterly 28 (1), S. 94-101 Plantenga, J./Remery, C. (2008): The Provision of Childcare Services in the Netherlands, External report commissioned by and presented to the EU Directorate-General Employment and Social Affairs, Brüssel

Plasman, R. (2008): The Provision of Childcare Services in Luxembourg, External report commissioned by and presented to the EU Directorate-General Employment and Social Affairs, Brüssel

Roberts, H. (2000): What is Sure Start?, in: Archives of Disease in Childhood 82 (6), S. $435-437$

Sarti, R. (2010): Who cares for me? Grandparents, Nannies and Babysitters Caring for Children in Contemporary Italy, in: Paedagogica Historica 46 (6),

S. $789-802$

Schweizerische Konferenz der Kantonalen Erziehungsdirektoren (EDK) (2007): Schweizer Beitrag für die Datenbank Eurybase - The database on education systems in Europe

Seils, E. (2014): Die Vermessung des Wohlfahrtsstaates, in: Seils, E./Bogedan, C./Leiber, S. (Hrsg.): Sozialversicherung: Wandel, Wirkung, Weiterentwicklung, Wiesbaden (im Erscheinen)

Simonazzi, A. (2008): The Provision of Childcare Services in Italy, External report commissioned by and presented to the EU Directorate-General Employment and Social Affairs, Brüssel

Skolverket (2009): Children, pupils and staff. National level, Stockholm Skolverket (2011): Facts and Figures 2011. Pre-school activities, school-age childcare, schools and adult education in Sweden, Stockholm

Smith, R./Poole, E./Perry, J./Wollny, I./Reeves, A. (2010): Childcare and early years survey of parents 2009: National Centre for Social Research, London

Statistics Denmark (2012): Statistical Yearbook, Copenhagen

Statistics Norway (2012): StatBank. Kindergartens - level 2 (M) by region, contents and time, http://www.ssb.no/en/forside;jsessionid=05A4D2C393C4A3 E9ACE1655FD5D3FF5A.kpld-as-prod10

Statistik Austria (2013): Kindertagesheimstatistik, Wien

Sutela, H. (2008): The Provision of Childcare Services in Finland, External report commissioned by and presented to the EU Directorate-General Employment and Social Affairs, Brüssel

\section{AUTOREN}

ERIC SEILS, Dr., ist Wissenschaftler im Wirtschafts- und Sozialwissenschaftlichen Institut (WSI) in der Hans-Böckler-Stiftung. Arbeitsschwerpunkte: Vergleichende Wohlfahrtsstaatsforschung, Armut \& Soziales.

eric-seils@ boeckler.de

MATTHIAS KLEIN ist Volkswirt und Politikwissenschaftler. Arbeitsschwerpunkte: Sozialstaatsentwicklung in der Bundesrepublik.

matthias.klein@posteo.de 